\title{
SITUACIÓN ACTUAL DE LOS CONOCIMIENTOS SOBRE LA PALEOFLORA DE LA COMUNIDAD DE MADRID
}

\author{
Concepción ÁLVAREZ RAMIS ${ }^{1}$ y Teresa FERNÁNDEZ MARRÓN ${ }^{1}$ \\ 1 Departamento y U.E.I. de Paleontología. Facultad de Ciencias Geológicas (UCM) e Instituto de Geología Económica (CSIC). \\ 28040 Madrid.
}

\begin{abstract}
Álvarez Ramis, C. y Fernández Marrón, T. 1996. Situación actual de los conocimientos sobre la paleoflora de la comunidad de Madrid. [Current knowledge on the palaeoflora of the Madrid community]. Revista Española de Paleontologia, N" Extraordinario, 190-194. ISSN 0213-6937.
\end{abstract}

\begin{abstract}
Paleobotanic studies carried out in the framework of several projects, as well as others carried out with foreign paleobotanic research centres of France (Strasbourg, Lyon, Marseille, Paris) and Hungary (Szeged) are summarized.

The results obtained in the four research lines approached (Paleohistology, Palynology, Paleochemistry and Macroflora) are resumed and an assessment is made on the chronology and the environmental aspects of the Upper Cretaceous and Miocene of the Madrid province.
\end{abstract}

Keywords: Paleobotany, Palynology, Paleohistology, Paleochemistry, Cretaceous, Miocene, Madrid.

RESUMEN

Se enumeran los trabajos paleobotánicos realizados en el marco de una serie de proyectos así como otros coordinados con centros de investigación paleobotánica de Francia (Estrasburgo, Lyon, Marsella y Paris) y Hungría (Szeged).

Se sintetizan los resultados obtenidos en las cuatro líneas de investigación abordadas (Paleohistología, Palinología, Paleobioquímica y macroflora) y se especula sobre la cronología y medioambiente durante el Cretácico superior y el Mioceno en la Comunidad de Madrid.

Palabras clave: Paleobotánica, Palinología, Paleohistología, Paleobioquímica, Cretácico, Mioceno, Madrid..

\section{INTRODUCCIÓN}

En este trabajo intentamos sintetizar los resultados de las investigaciones que hemos realizado, en Madrid y su provincia, en el marco de 24 proyectos de investigación patrocinados más o menos directamente por el CSIC y en menor escala por el Ayuntamiento y la Comunidad de Madrid.

En la realización de nuestros estudios hemos contado con la valiosa colaboración de especialistas, nacionales y extranjeros, en varias disciplinas complementarias de la Paleobotánica.

El núcleo del equipo que llevó a cabo estos proyectos lo integraban profesores, investigadores y colaboradores de la Facultad de Ciencias Geológicas de la UCM, del Instituto de Geología Económica y del Instituto de Ciencias Medioambientales del CSIC. En este equipo se fueron integrando, a medida que las circunstancias lo aconsejaron, investigadores procedentes de centros punteros relacionados con la Paleobotánica.

Como resultado de estas investigaciones han surgido una serie de datos, conocimientos, métodos y técnicas de interés en aplicación geológica.

Este trabajo incluye los estudios paleobotánicos efectuados sobre materiales postpaleozoicos procedentes de la Comunidad de Madrid.

Las dos áreas de estudio se delimitan claramente. En el Cretácico del Borde Sur de la Sierra de Guadarrama al Norte de la Comunidad de Madrid, se localizan los ocho yacimientos estudiados en profundidad y otros cinco en los que se han iniciado estudios.

Los yacimientos cretácicos se agrupan en torno a dos núcleos de población. Unos incluidos dentro de una circunferencia de $12 \mathrm{~km}$. de radio, cuyo centro es el pueblo de Guadalix de la Sierra y otros, en el círculo cuyo centro lo ocupa el pueblo de Torrelaguna.

Los estudios sobre flora cretácica fueron realizados en el marco de ocho proyectos nacionales y trece proyectos internacionales que, concatenados con los nacionales, amplían algunos de los temas de mayor interés.

Al Sur de la Comunidad, en la fosa del Tajo, se encuentran los yacimientos terciarios estudiados por la autoras desde el punto de vista paleobotánico. Estos materiales proceden de sondeos, realizados en la sucesión evaporítica cenozoica del Sur de Madrid. Los yacimientos estudiados se ubican en la capital de España, uno de ellos en el barrio de Portazgo y el otro en Cerro Negro, al pie de los barrios de La Viña y La Paz. En ambos sólo puede ser estudiada la Paleobotánica desde el punto de vista micropaleontológico, pués prácticamente no existen macrorrestos completos. Los palinomorfos son muy abundantes y variados y están en perfecto estado de preservación, permitiendo el estudio del querógeno.

Las investigaciones sobre paleoflora cenozoica fueron costeadas parcialmente por el IGME, el Ayuntamiento y la 
Comunidad de Madrid y han dado lugar, hasta el momento, a cuatro publicaciones (Alvarez Ramis y Fernández Marrón 1984; Almendros et al. 1988; Alvarez Ramis y Fernández Marrón, 1993 y Alvarez Ramis, en prensa).

Dado que los aspectos relativos a geología regional y que las sucesiones litoestratigráficas establecidas, en cada uno de los cortes o sondeos a los que se hace referencia, han sido ya dadas a conocer en varias publicaciones así como por el escaso espacio de que se dispone, no insistimos estos aspectos.

\section{ASPECTOS PALEOBOTÁNICOS ABORDADOS}

Los antecedentes paleobotánicos en la provincia de Madrid son casi inexistentes. La primera cita referida a macroflora del Cretácico, corresponde a Menéndez Amor (1952). Existen otras indicaciones de relativo interés en publicaciones no paleobotánicas (Arias Ordás, 1969 y Corchón, 1976).

A partir de 1978, en que dimos a conocer los primeros resultados paleobotánicos parciales, se han publicado otros de forma ininterrumpida que nos permiten hoy sacar conclusiones de conjunto. En los distintos proyectos se abordaron fundamentalmente aspectos concernientes a megaflora, microflora, paleohistología y paleobioquímica que incluyen distintas temáticas que van desde el clásico estudio morfológico de restos macroscópicos, principalmente foliares, al estudio palinológico pasando por el conocimiento de la anatomía interna de las plantas que por su tipo de fosilización y caracteristicas lo permitieron y la determinación paleobioquímica del contenido de los querógenos.

Marginalmente se abordaron otros aspectos consecuentes al análisis de los resultados.

A partir de 1980 se está estudiando la composición de la materia orgánica de los restos fósiles momificados hallados en los lignitos; así como la contenida en las calcarenitas y margas de procedencia de los vegetales fósiles.

De entre la macroflora se seleccionaron fragmentos de Frenelopsis oligostomata, que conservaban en parte su composición original, y restos de troncos de Coníferas en las que se podía reconocer aún su anatomía interna. Los primeros datos publicados sobre los análisis paleobioquímicos de estos restos vegetales procedían del yacimiento de Torrelaguna (Almendros et al., 1982). Un estudio similar se efectuó, en el mismo año, en tejidos epidérmicos procedentes de yacimientos próximos al embalse de El Vellón. (Alvarez Ramis et al., 1982).

\section{RESULTADOS Y CONCLUSIONES OBTENIDOS EN EL CRETÁCICO SUPERIOR DEL BORDE SUR DE LA SIERRA DE GUADARRAMA}

Se resumen a continuación los resultados y conclusiones logrados durante los casi veinte años dedicados a estudiar la flora cretácica de la provincia de Madrid.

\section{Resultados}

En la primera década (1978-88) nuestras investigaciones se centraron en el estudio de las canteras denominadas Torrelaguna, Los Alcores y El Vellón-1, comenzando por los estudios litológicos, establecimiento de secuencias litoestratigráficas y localización de restos paleobotánicos. Se pusieron de manifiesto, en disposición más o menos cíclica, algunos niveles de calizas muy compactas que presentaban moldes de hojas y ramas de gran vistosidad pero de poca importancia para el investigador por la dificultad de interpretación, y otros niveles carbonosos, de escasa potencia, con una rica microflora y restos de macroflora muy fragmentada, de gran interés paleobotánico.

La macroflora cretácica fué objeto de estudios a partir de 1975. Los primeros datos obtenidos se dieron a conocer en la "Première Table Ronde sur les Ecosystèmes continentaux du Mésozoïque", celebrada en Paris en 1978. Una puesta al día de los conocimientos sobre macroflora cretácica de la provincia de Madrid se da a conocer posteriormente (Álvarez Ramis, 1980 y Álvarez Ramis, 1981b).

Gómez Porter (1983) en su Tesis de Licenciatura, estudió los macrorrestos vegetales procedentes de dos yacimientos próximos al embalse de "El Vellón", describiendo una serie de niveles muy ricos en macroflora. Posteriormente se profundiza en el conocimiento de todos los megarrestos de las sucesiones litoestratigráficas de estas canteras, llegando a precisar más de cincuenta especies-forma entre las que dominaban, en número de restos, las Coníferas y en variedad las Dicotiledóneas (Álvarez Ramis et al., 1984).

La mayoría de los macrorrestos hallados en estas canteras corresponden a morfotipos de Coníferas de los géneros Frenelopsis, Araucaria, Sequoia, y Taxodium, y en menor número se encuentran las Angiospermas aunque son muy variadas en especies de forma, sobresaliendo las Myricáceas.

Algunas plantas fósiles conservaban "in situ" parte de sus cutículas, y sobre ellas se realizaron posteriormente estudios paleohistológicos y paleobioquímicos.

El hallazgo de macrorrestos de los aparatos vegetativo y reproductor de ciertas plantas permitió hacer su reconstrucción y con posterioridad, al estudiar sus palinomorfos, anatomía interna y caracteristicas cuticulares, su reconocimiento sistemático. De esta forma se logró el reconocimiento integral de la Conífera Frenelopsis oligostomata de la que conocemos ya todos sus órganos excepto el reproductor femenino (macrocono). Esta Conífera se encuentra en todos los yacimientos estudiados, apareciendo sus restos foliares, tallos y pólenes con mucha frecuencia (Álvarez Ramis, 1981a; Álvarez Ramis et al., 1982; Álvarez Ramis et al., 1987 y Barale et al., 1988).

Se analizó también el polimorfismo presente en los restos de Frenelopsis y las coincidencias morfológicas existentes entre las formas de $F$. alata y $F$. oligostomata por si las plantas clasificadas en Torrelaguna como $F$. oligostomata correspondieran en realidad a dos especies. El análisis paleohistológico y paleobioquímico no ponen de manifiesto entre estas plantas ninguna diferencia esencial (Álvarez Ramis et al., 1984).

En el yacimiento de los Alcores hemos encontrado varios restos foliares del género Dalbergia que hemos podido llegar a clasificar mediante el estudio de sus epidermis y 
en parte por su identidad morfológica con el paragénero Dalbergites (Álvarez Ramis, 1985b)

Los lignitos nos proporcionaron el material más interesante, al permitir efectuar estudios paleohistológicos y paleobioquímicos, al margen del reconocimiento integral de una serie de vegetales fósiles (Frenelopsis oligostomata, Dalbergia sp. y Weichselia reticulata entre otros).

En la presente década, sin descartar otros aspectos, nuestros esfuerzos se están centrando básicamente en los estudios micropaleontológicos, especialmente en el reconocimiento e interpretación de las paleoasociaciones de Normapólenes, al ser fundamentales en las correlaciones de sedimentos continentales del tránsito Cretácico-Terciario y en determinaciones cronoestratigráficas y paleobiogeográficas.

El análisis comparativo entre el estudio de los macrorrestos y microrrestos permitió el reconocimiento de varios grupos de Pteridófitos incluidos en diversos taxa de Licopodiales, Selaginelales, Equisetáceas y Filicales (Álvarez Ramis y Gómez Porter, 1990)

A partir de 1991 se continuaron estudios en otros yacimientos de la zona y/o se completaron aspectos no abordados en los yacimientos ya estudiados.

Álvarez Ramis et al. (1992) dieron a conocer los resultados obtenidos a partir del estudio de numerosos fragmentos de ramas que se descubrían en un corte de la secuencia del Barranco de Patones. Las plantas, que en su mayoría se presentaban bajo la forma de impresiones, correspondían a ramas de Coníferas de hoja microscópica. Algunas presentaban, "in situ", macro y microconos permitiendo clasificarlas como Glyptostrobus sp.

Continuamos aún el estudio paleohistológico de todos aquellos restos fósiles que conservan parte de sus componentes orgánicos.

Con posterioridad al estudio cuticular de los restos de Frenelopsis y Dalbergia ya citados, nuestros esfuerzos se encaminan al reconocimiento de dos especies del género Araucaria (Álvarez Ramis, 1985a) ya que se han hallado dispersos dos tipos de pólenes, Araucariacites australis y A. hungaricus(Álvarez Ramis y Doubinger, 1994). Se estudian las epidermis de algunas especies actuales de Araucaria, dadas las semejanzas morfológicas entre estas especies y algunos de los macrorrestos fósiles estudiados. Otro dato a tener en cuenta en este sentido es la presencia de pequeños granos de copal, posiblemente debida a resinas segregadas por las hojas de una especie fósil próxima a $A$. araucana (Álvarez Ramis y Fernández Marrón, 1992).

Los fósiles más abundantes e interesantes de las series del Cretácico de la provincia de Madrid son los palinomorfos al encontrarse, en mayor o menor proporción, en casi todos los paquetes.

Los estudios palinológicos del Cretácico han puesto de manifiesto resultados muy interesantes en Cronoestratigrafía como el hecho de que las proporciones relativas entre los distintos taxa de una paleoasociación puedan considerarse biomarcadores. La variedad de formas de Angiospermas y entre ellas la dominancia de ciertos tipos de Normapólenes indican que la génesis de los niveles que los incluyen tuvo lugar en las cercanías del Terciario.

En estos últimos años queremos intensificar las investigaciones palinológicas, al constatar que estas nos proporcionan datos y conclusiones de aplicación práctica.

\section{Conclusiones cronoestratigráficas}

El análisis de los datos aportados por los estudios paleobotánicos realizados sobre diversos tipos de materiales cretácicos del Borde Sur de la Sierra de Guadarrama, parece apuntar hacia una edad más reciente que las supuestas en un estudio hidrogeológico de la zona (Corchón, 1976). Este autor atribuye una edad Cenomaniense al yacimiento de Torrelaguna y Turoniense a los yacimientos situados más al Oeste.

La primera datación correspondió al yacimiento de Torrelaguna. Su edad se estimó en base a la composición palinológica (Álvarez Ramis y Doubinger, 1980). Las autoras indican que el yacimiento podía haberse formado entre el Cenomaniense superior y el Santoniense, aunque la presencia de formas atribuidas a "Piolencipollis piolensis" y Traslucentipollenites fsp. apunten al menos al Campaniense. El hecho de que estos pólenes se presenten en bajos porcentajes incluso puede deberse a resedimentación.

La cantera denominada "Las Calerizas", situada en la carretera que une el pueblo de Guadalix de la Sierra con el de Colmenar Viejo, es rica en palinomorfos (Medus y Álvarez Ramis, 1989). El espectro polínico presenta un elevado porcentaje de Normapólenes, dominando Interporopollenites fsp. y diversidad de otras formas de Angiospermas, que están presentes a partir del Campaniense. Los autores especulan también sobre la posibilidad de que la paleoasociación hallada hubiese sido modificada por factores climáticos, fitogeográficos, ecológicos, sedimentológicos, etc.

Durante el 9. ${ }^{\circ}$ Simposio de Palinología se propuso, para el yacimiento del Cerro de la Mesa próximo a Guadalix de la Sierra, en base a las paleoasociaciones esporopolínicas halladas, una edad Maastrichtiense (Alvarez Ramis et al., 1992).

Recientemente Álvarez Ramis y Doubinger (1994) indican, por la abundancia y variedad de los Normapólenes y en particular de formas del género Interporopollenites y Papillopollis , así como la presencia de Subtriporopollenites fsp., Ilexpollenites fsp.,Polyporopollenites fsp. Cupuliferopollenites fsp. y diversos tipos de pólenes inaperturados, que el yacimiento de "Los Alcores", próximo al embalse de El Vellón, se formó en el intervalo Campaniense superior-Maastrichtiense.

Los datos aportados por el estudio de los megarrestos vegetales son coincidentes con los de la microflora en lo que a Cronoestratigrafía se refiere.

\section{Conclusiones paleogeográficasy paleoambientales}

El conjunto de la paleoflora reconocida en el Cretácico final del Borde Sur de la Sierra de Guadarrama, la presencia de dinoquistes y de cámaras internas de Foraminíferos en algunos niveles (Álvarez Ramis y Doubinger, 1980 y 1994) nos determinan una posición más o menos margino-litoral y evidencian un clima cálido y seco a excepción de las zonas beneficiadas por la evaporación de las aguas costeras y lagunas interiores condicionantes de que se acumularan, en las áreas de sedimentación fitógena, plantas de hábitats muy diferentes.

La morfología foliar, especialmente algunos rasgos (borde, nerviación, grado de cutinización y tamaño) son de gran utilidad en la determinación de hábitats, distribución, clima etc. (Álvarez Ramis, 1984).

Hemos hallado restos de plantas con hojas de pequeñas dimensiones (en algunos casos vestigiales), de bordes recor- 
tados (a veces espinosos) y de limbos coriáceos. Tipos foliares de estas características se dan en altos porcentajes en las zonas xerofíticas y/o más o menos áridas. Estas características las presentan entre otras plantas mesozoicas las Cheirolepidiáceas, Myricáceas y Weichselia reticulata.

Junto con las morfologías foliares indicadas aparecen también restos de gran superficie foliar, márgenes lisos, cima apuntada y nerviaciones de tipo camptódromo o broquídromo. Estas morfologías en oposición a las citadas en el párrafo anterior, se presentan en altos porcentajes en zonas cálidas y húmedas. Algunos restos de estas características están presentes en casi todos los yacimientos. (Álvarez Ramis 1980, 1981b, 1985a; Álvarez Ramis et al., 1984).

Es factor constante, en todos los niveles y yacimientos cretácicos estudiados, la presencia de la familia Cheirolepidiáceas representada por fragmentos del aparato vegetativo y/o reproductor de Frenelopsis oligostomata (Álvarez Ramis, 1981a). En algunos niveles los macrorrestos de Frenelopsis son más bien escasos, aunque normalmente se presentan paritarios con los de las otras Coníferas. Excepcionalmente existen pequeños niveles de lignitos formados casi exclusivamente por restos de esta Conífera.

Frenelopsis oligostomata (sus pólenes son conocidos como Classopollis classoides) es planta eurihalina de amplia distribución durante el Mesozoico y que en asociación con el helecho Weichselia reticulata constituía la base de la vegetación de los paleomanglares pantropicales del Tethys y Preatlántico. Las plantas de Frenelopsis remontaban las desembocaduras y zonas pantanosas varios kilometros tierra adentro.

Los macrorrestos del helecho eurihalino Weichselia reticulata hallados en el Cretácico de Madrid son escasos y muy fragmentarios, si bien varios aparecen en aceptable estado de conservación (Álvarez Ramis y Gómez Porter, 1990).

La desproporción que existe entre los escasos fósiles hallados de Weichselia y la relativa abundancia de los de Frenelopsis, parece evidenciar que en la provincia de Madrid durante el Cretácico superior no perduraban las formaciones de paleomanglares pero la persistencia de algunas plantas de Weichselia atestiguan su existencia anterior.

\section{FLORA TERCIARIA EN LA PROVINCIA DE MADRID}

La paleoflora terciaria ha sido abordada con menor intensidad que la mesozoica. Los aspectos paleobotánicos que se abordan en los proyectos sobre flora cenozoica fueron los mismos que se tuvieron en cuenta para la flora cretácica (megaflora, palinología, paleohistología y paleobioquímica).

Los estudios concernientes a macroflora terciaria prácticamente no se desarrollaron, sobre todo por la escasez de restos de hojas y ramas. Tampoco realizamos estudios paleohistológicos como consecuencia de la pobreza en macrorrestos y por ello la escasez y deterioro de las cutículas así como por pérdida de su anatomía interna.

Los estudios paleobioquímicos rindieron datos de interés y fueron consecuentes con los resultados aportados por el análisis de los palinomorfos, únicos restos paleobotánicos que se pudieron estudiar en las series neógenas del Sur de Madrid.

A medida que se fueron obteniendo datos se dieron a conocer los resultados. En 1983 y 1985 se entregaron, al coordinador de los proyectos, sendos informes que englobaban uno de ellos, los resultados y conclusiones de conjunto de varios yacimientos del Sureste de Madrid y el segundo los del yacimiento de Portazgo.

Al margen de estos dos informes se elaboraron varias publicaciones. En la primera de ellas se da a conocer un estudio palinológico preliminar efectuado en materiales procedentes de un nivel localizado en Cerro Negro (Madrid) que presentaba una microflora muy interesante (Álvarez Ramis y Fernández Marrón, 1984).

Con posterioridad (Almendros et al., 1988) dan a conocer el estudio paleobioquímico del querógeno procedente de tres niveles, ricos en materia orgánica, de un sondeo que había sido efectuado con anterioridad por el Servicio Geológico de Obras Públicas en el barrio de Portazgo (Madrid). Los autores delimitan un hábitat pantanoso, con un cierto grado de oxigenación, en el que se encontraban numerosas plantas continentales en descomposición y junto a ellas un número relativamente importante de organismos inferiores entre los que predominaban los quistes de Dinoflagelados.

Posteriormente se amplió el estudio de este sondeo, elevando de tres a nueve los niveles objeto de análisis palinológico (Álvarez Ramis y Fernández Marrón, 1993). En esta publicación sólo se dieron a conocer estos niveles, a pesar de que se analizaron treinta y dos, dado que los restantes presentaban muy baja densidad en palinomorfos por lo que no se pudo sacar conclusiones de ellos y su estudio quedó relegado para fechas posteriores. Las muestras de los niveles analizados no fueron tomadas a intervalos regulares sino de acuerdo con la riqueza en materia orgánica que presentaban los tramos elegidos de la serie local.

Dado el interés que mostraba el yacimiento de Portazgo se continuó con el estudio de los nueve niveles citados.

En Septiembre de 1993 C. Álvarez Ramis, con motivo de pronunciar una conferencia en la Birbal Savitri Sahni Foundation de Lucknow (India), dió a conocer en profundidad las distintas paleobioasociaciones halladas en estos nueve niveles, que habian sido determinadas básicamente teniendo en cuenta el estudio palinológico.

En la conferencia se puso de manifiesto que los espectros polínicos, hallados en los sucesivos niveles estudiados a lo largo de la secuencia litoestratigráfica de Portazgo, no representan cambios de importancia en la vegetación de la Cuenca del Tajo durante el Neógeno, si bien se detectan algunas variaciones ecológicas de acuerdo con la profundidad y grado de colmatación de la zona lacustre.

Cronoestratigráficamente la secuencia, puesta de manifiesto por el sondeo, se integraría en el intervalo Aragoniense-Vallesiense inferior, sin poder descartar para los niveles más inferiores un grado de proximidad más estrecho con el Paleógeno.

El Instituto de Paleobotánica "Birbal Sahni" de Lucknow, a su vez, se interesó en el tema de la conferencia y solicitó la elaboración de una monografía, que se encuentra en vias de publicación, como Memoria temática de dicho Instituto (Álvarez Ramis, en prensa).

\section{AGRADECIMIENTOS}

Esta publicación se incluye en el proyecto PB-92/0101 de la DGICYT. 


\section{BIBLIOGRAFÍA}

Almendros, G. Álvarez Ramis, C y Polo, A. 1982. Estudio paleobioquímico de la materia orgánica de restos fosilizados de Frenelopsis procedentes del Supracretácico de Torrelaguna (Madrid). Revista de la Real Academia de Ciencias Exactas, Fisicas y Naturales de Madrid, LXXVI, 285-302

Almendros, G. González Vila, F., Martín, F. and Álvarez Ramis, C., 1988. Structural studies on Miocene kerogen. Fuel, 67, 502-507.

Álvarez Ramis, C. 1980. Sur la macroflore du Crétacé continental de l'Espagne. Memoires Société Géologique de France. N.S. 139, 5-9.

Álvarez Ramis,C. 1981a. Estudio de Frenelopsis oligostomata (Romariz) Alvin, procedente del Cretácico superior de Torrelaguna (Madrid). Estudios Geológicos, 37, 83-87

Álvarez Ramis,C. 1981b. Les Macroflores de Crétacé Moyen continental de l'Espagne in: Les végétaux (macrofossiles) du Crétacé Moyen de l'Europe Occidentale et du Sahara. Végétations et Paléoclimats (Alvarez Ramis, Biondi, Desplats, Hughes, Koeninger, Pons et Rioult). Cretaceous Research, 2, 339-352.

Álvarez Ramis, C. 1984. La biometría foliar en la determinación de paleoclimas terciarios. Libro Jubilar de J.M. Rios. III, 137-145.

Álvarez Ramis, C. 1985a. Sur la paléoecologie des alentours du Barrage "El Vellón" (Madrid, Espagne) pendant le Crétacé supérieur. in: Paléobotanique. Bulletin des Sciences (M.E.N.) 5, 115-125.

Álvarez Ramis, C. 1985b. Sur l'intérêt des restes d'Angiospermes provenant du Crétacé supérieur de la Bordure Sud de la Sierra de Guadarrama (Province de Madrid, Espagne).in: Paléobotanique, Recherches nouvelles sur l'évolution végétale. Volume Jubilaire en l'honneur du Prof Boureau. Bulletin des Sciences.(M.E.N.), 8, 153-164

Álvarez Ramis, C.(en prensa). The palaeoflora obtained of a drilling cross over the main lithostratigraphic unit of Southern Madrid (Spain). Memories Birbal Sahni Institute of Palaeobotany. Lucknow (India).

Álvarez Ramis, C. Almendros, G. et Polo, A. 1982. Sur la présence du genre Frenelopsis dans le gisement de Guadalix de la Sierra (Madrid, Espagne) in: Paléobotanique. Bulletin des Sciences (M.E.N.) Paris, I,85-95.

Álvarez Ramis, C., Almendros, G. y Polo, A. 1984. Precisiones histológicas y bioquímicas acerca de los ejemplares de Frenelopsis procedentes de Torrelaguna (Madrid). Estudios Geológicos, 40, 107-113.

Álvarez Ramis, C. Barale, G. et Fernández Marrón, T. 1987. Nouvelles observations au Microscope électronique à balayage sur la cuticule de Frenelopsis oligostomata Romariz emend. Alvin 1977, du Crétacé supérieur de Torrelaguna (province de Madrid) in: Paléobotanique. Bulletin des Sciences (M.E.N.) Paris, II;, 67-74.

Álvarez Ramis, C. y Doubinger, J. 1980. Los microfósiles vegetales del Cretácico superior de Torrelaguna (Madrid). Boletín Real Sociedad Española de Historia Natural (Geología), 78, 205-215.

Álvarez Ramis, C. y Doubinger, J. 1994. Contribución al conocimiento de la palinoflora cretácica de la provincia de
Madrid.Los Alcores, Guadalix de la Sierra. Revista Española de Micropaleontología, XXVI, 5-22.

Álvarez Ramis, C. y Fernández Marrón, T. 1984. Consideraciones en torno a la microflora de un nivel correspondiente a la serie miocénica del Municipio de Madrid. in: Memoria II Congreso Latinoamericano de Paleontología. Oaxtepec (Méjico), 509-515.

Álvarez Ramis, C. et Fernández Marrón, T. 1992. Essai de détermination génerique de restes fossiles considerés comme Araucariacées. OFP informations. $\mathrm{N}^{\circ}$ Spécial 16B, 4

Álvarez Ramis, C. et Fernández Marrón, T. 1993. Étude micropaleontologique d'echantillons provenant du Sud de la Ville de Madrid. OFP informations, 18, 9

Álvarez Ramis, C. Fernández Marrón, T. et Gómez Porter, P. 1984. Étude des niveaux a plantes du Crétacé supérieur d'une carrière située au Bord du Barrage "El Vellón" (Madrid, Espagne).in : Paléobotanique. Bulletin des Sciences (M.E.N.).Fasc. II, 83-97.

Álvarez Ramis, C. Fernández Marrón, T, y Gomez Porter, P. 1992. Presencia de restos de Glyptostrobus próximos a la especie actual G. lineatus (Poiret) Druce en niveles terminales de la serie supracretácica del Borde Sur de la Sierra de Guadarrama. Barranco de Patones (Madrid). in: Actas III Congreso Geológico de España y VIII Congreso Latinoamericano de Geología. Salamanca, I, 483-486.

Álvarez Ramis,C. y Gómez Porter,P. 1990. Estudio palinológico de Pteridófitos del Cretácico superior de la provincia de Madrid: 1. " parte:Embalse de "El Vellón". Acta salmaticensia 68, 4755. Salamanca.

Álvarez Ramis, C. Kedves, M. y Fernández Marrón, T. 1992. Asociaciones esporopolínicas del Cretácico superior del Cerro de la Mesa (Guadalix de la Sierra, Madrid). Resumenes $9^{\circ}$ Simposio de Palinología APLE. Las Palmas de Gran Canaria, 28.

Arias Ordás, C. 1969. Estudio estratigráfico y sedimentología del Cretácico de los alrededores de Guadalix de la Sierra. Cuadernos de Geología Ibérica. 1, 309-333.

Barale, G., Fernandez Marrón, T. et Alvarez Ramis, C. 1988. Étude de cônes mâles de Frenelopsis Schenk emend.Reymanowna \& Watson, 1976 (Cheirolepidiaceae) du Crétacé superieur de Torrelaguna (Province de Madrid, Espagne). Geobios, 21, 2, 187-199. Lyon

Corchón, F. 1976. Estudio hidrogeológico del Cretácico de los alrededores de Torrelaguna (Madrid y Guadalajara). Informaciones y Estudios. Servicio Geológico. M.O.P. 40, 189p.

Gómez Porter, P. 1983. Estudio paleobotánico de los macrorrestos de dos canteras sitas en el borde Sur del embalse de El Vellón (Guadalix de la Sierra, Madrid). Memoria de Licenciatura. Facultad de Ciencias Geológicas. UCM, 115 p.

Médus, J. et Alvarez Ramis, C. 1989. Des pollens Normapolles d'un niveau du Crétacé superieur de la region de Guadalix, Prov. de Madrid, Espagne. Revista Española de Micropaleontología 21, 1, 139-144.

Menéndez Amor, J. 1952. Un nuevo yacimiento de plantas fósiles. Boletín Real Sociedad Española de Historia Natural. 50, 131-132.

Manuscrito recibido: 15 de diciembre, 1994 Manuscrito aceptado: 24 de mayo, 1995 